\title{
Double-Diffusive Convection in Compressible Viscoelastic Dusty Fluid Through Brinkman Porous Media
}

\author{
Pardeep Kumar*, Hari Mohan
}

Department of Mathematics, ICDEOL, Himachal Pradesh University, Shimla, 171005, India

\begin{abstract}
The double-diffusive convection in compressible Rivlin-Ericksen viscoelastic fluid with suspended particles through Brinkman porous medium is considered. Following the linearized stability theory and normal mode analysis, the dispersion relation is obtained. For stationary convection, the Rivlin-Ericksen viscoelastic fluid behaves like a Newtonian fluid and it is found that suspended particles and medium permeability have destabilizing effect whereas stable solute gradient and compressibility have stabilizing effect on the system. Graphs have been plotted by giving numerical values to the parameters to depict the stability characteristics. The stable solute gradient and viscoelasticity are found to introduce oscillatory modes in the system which is non-existent in their absence.
\end{abstract}

Keywords Double-Diffusive Convection, Compressible Rivlin-Ericksen Viscoelastic Fluid, Brinkman Porous Medium, Suspended Particles

\section{Introduction}

The theoretical and experimental results of the onset of thermal instability (Bénard convection) in a fluid layer under varying assumptions of hydrodynamics has been treated in detail by Chandrasekhar[1] in his celebrated monograph. The problem of thermohaline convection in a layer of fluid heated from below and subjected to a stable salinity gradient has been considered by Veronis[2]. The physics is quite similar in the stellar case in that helium acts like salt in raising the density and in diffusing more slowly than heat. The conditions under which convective motions are important in stellar atmospheres are usually far removed from consideration of a single component fluid and rigid boundaries, and therefore it is desirable to consider a fluid acted on by a solute gradient and free boundaries. The problem of double diffusive convection in fluids through porous medium is of importance in geophysics, soil sciences, groundwater hydrology and astrophysics. The development of geothermal power resources holds increased general interest in the study of the properties of convection in porous medium. The scientific importance of the field has also increased because hydrothermal circulation is the dominant heat transfer mechanism in the development of young oceanic crust (Lister[3]). Generally it is accepted that comets consists of a dusty "snowball" of a mixture of frozen gases which, in the process of their journey, changes from solid to gas and vice-versa. The physical properties of comets,

* Corresponding author:

pkdureja@gmail.com (Pardeep Kumar)

Published online at http://journal.sapub.org/ajfd

Copyright (C) 2012 Scientific \& Academic Publishing. All Rights Reserved meteorites and interplanetary dust strongly suggest the importance of porosity in the astrophysical context. Mounting evidence, both theoretical and experimental, suggests that Darcy's equation provides an unsatisfactory description of the hydrodynamic conditions, particularly near the boundaries of a porous medium. Beavers et al[4] have experimentally demonstrated the existence of shear within the porous medium near surface, where the porous medium is exposed to a freely flowing fluid, thus forming a zone of shear- induced flow field. The Darcy's equation however, cannot predict the existence of such a boundary zone, since no macroscopic shear term is included in this equation (Joseph and $\mathrm{Tao}[5])$. To be mathematically compatible with the Navier-Stokes equations and physically consistent with the experimentally observed boundary shear zone mentioned above, Brinkman proposed the introduction of the term $\frac{\mu}{\varepsilon} \nabla^{2} \vec{q}$ in addition to $-\left(\frac{\mu}{k_{1}}\right) \vec{q}$ in the equations of fluid motion. Stommel and Fedorov[6] and Linden[7] have remarked that the length scales characteristic of double-diffusive convecting layers in the oceans could be sufficiently large for Earth's rotation to become important in their formation. Moreover, the rotation of the Earth distorts the boundaries of a hexagonal convection cell in a fluid through a porous medium, and the distortion plays an important role in the extraction of energy in the geothermal regions. Brakke[8] explained a double-diffusive instability that occurs when a solution of a slowly diffusing protein is laid over a denser solution of more rapidly diffusing sucrose. Nason et al[9] found that this instability, which is deleterious to certain biochemical separations, can be suppressed by rotation in the ultracentrifuge.

In geophysical situations, the fluid is often not pure but contains suspended particles. Scanlon and Segel[10] have 
considered the effects of suspended particles on the onset of Be'nard convection and found that the critical Rayleigh number is reduced because of the heat capacity of the particles. The suspended particles were thus found to destabilize the layer. Palaniswamy and Purushotham[11] have studied the stability of shear flow of stratified fluids with fine dust and found the effects of fine dust to increase the region of instability

The use of the Boussinesq approximation has been made throughout, which states that variations of density in the equations of motion can safely be ignored everywhere except in its association with the external force. The approximation is well justified in the case of incompressible fluids. When the fluids are compressible, the equations governing the system become quite complicated. To simplify them, Boussinesq tried to justify the approximation for compressible fluids when the density variations arise principally from thermal effects. Spiegel and Veronis'[12] have simplified the set of equations governing the flow of compressible fluids under the following assumptions:

(a) the depth of the fluid layer is much less than the scale-height, as defined by them;

and (b) the fluctuations in temperature, density and pressure, introduced due to motion, do not exceed their total static variations.

There is growing importance of non-Newtonian fluids in geophysical fluid dynamics, chemical technology and petroleum industry. Bhatia and Steiner[13] have studied the problem of thermal instability of Maxwellian viscoelastic fluid in the presence of rotation and have found that the rotation has a destabilizing influence in contrast to the stabilizing effect on an ordinary viscous (Newtonian) fluid. The thermal instability of an Oldroydian viscoelastic fluid acted on by a uniform rotation has been studied by Sharma[14]. There are many elastico-viscous fluids that cannot be characterized by Maxwell's or Oldroyd's constitutive relations. The Rivlin-Ericksen elastico-viscous fluid is one such fluid. Rivlin and Ericksen[15] have studied the stress-deformation relaxations for isotropic materials. Polymers are used in the manufacture of spacecrafts, aeroplanes, tyres, belt conveyers, ropes, cushions, seats, foams, plastics engineering equipments, contact lens, etc. Rivlin-Ericksen viscoelastic fluid forms the basis for the manufacture of many such important and useful products. Thermal instability in Rivlin-Ericksen elastico-viscous fluid in presence of rotation and magnetic field, separately, has been investigated by Sharma and Kumar $([16,17])$. Sharma and Kumar[18] have studied the hydromagnetic stability of two Rivlin-Ericksen elasticoviscous superposed conducting fluids. Kumar and Singh[19] have studied the stability of two superposed Rivlin-Ericksen viscoelastic fluids in the presence of suspended particles. In another study, Kumar et al[20] have studied the hydrodynamic and hydromagnetic stability of two stratified Rivlin-Ericksen elastico-viscous superposed fluids.

Keeping in mind the importance in geophysics, astrophysics and various applications mentioned above, the double-diffusive convection in compressible Rivlin-Ericksen viscoelastic fluid with suspended particles through Brinkman porous medium has been considered in the present paper.

\section{Formulation of the Problem and Perturbation Equations}

Here we consider an infinite horizontal, compressible Rivlin-Ericksen viscoelastic fluid-particle layer of thickness $\mathrm{d}$ bounded by the planes $\mathrm{z}=0$ and $\mathrm{z}=\mathrm{d}$ in a porous medium of porosity $\varepsilon$ and permeability $\mathrm{k}_{1}$. This layer is heated from below and subjected to a stable solute gradient such that steady adverse temperature gradient $\beta(=|d T / d z|)$ and a solute concentration gradient $\beta^{\prime}(=|d C / d z|)$ are maintained.

Let $\rho, \mu, \mu^{\prime}, p$ and $\vec{q}(u, v, w)$ denote respectively the density, viscosity, viscoelasticity, pressure and filter velocity of the pure fluid; $\overrightarrow{\mathrm{q}}_{\mathrm{d}}(\overline{\mathrm{x}}, \mathrm{t})$ and $\mathrm{N}(\overline{\mathrm{x}}, \mathrm{t})$ denote filter velocity and number density of the particles, respectively. If $g$ is acceleration due to gravity, $K=6 \pi \rho v \eta$ where $\eta$ is the particle radius, $\overrightarrow{\mathrm{q}}_{\mathrm{d}}=(1, \mathrm{r}, \mathrm{s}), \overline{\mathrm{x}}=(\mathrm{x}, \mathrm{y}, \mathrm{z})$ and $\vec{\lambda}_{1}=(0,0,1)$, then the equations of motion and continuity for Rivlin-Ericksen viscoelastic fluid permeated with suspended particles in a porous medium are

$$
\begin{aligned}
& \frac{\rho}{\varepsilon}\left[\frac{\partial \vec{q}}{\partial t}+\frac{1}{\varepsilon}(\vec{q} \cdot \nabla) \vec{q}\right]=-\nabla p-\rho g \vec{\lambda}_{1} \\
& +\left(\mu+\mu^{\prime} \frac{\partial}{\partial t}\right)\left(\frac{1}{\varepsilon} \nabla^{2}-\frac{1}{k_{1}}\right) \vec{q}+\frac{K N}{\varepsilon}\left(\vec{q}_{d}-\vec{q}\right), \\
& \left(\varepsilon \frac{\partial}{\partial \mathrm{t}}+\overrightarrow{\mathrm{q}} \cdot \nabla\right) \rho+\rho \nabla \cdot \overrightarrow{\mathrm{q}}=0 .
\end{aligned}
$$

Since the distances between particles are assumed to be quite large compared with their diameter, the interparticle relations, buoyancy force, Darcian force and pressure force on the particles are ignored. Therefore, the equations of motion and continuity for the particles are

$$
\begin{gathered}
\mathrm{mN}\left[\frac{\partial \overrightarrow{\mathrm{q}}_{\mathrm{d}}}{\partial \mathrm{t}}+\frac{1}{\varepsilon}\left(\overrightarrow{\mathrm{q}}_{\mathrm{d}} \cdot \nabla\right) \overrightarrow{\mathrm{q}}_{\mathrm{d}}\right]=\mathrm{KN}\left(\overrightarrow{\mathrm{q}}-\overrightarrow{\mathrm{q}}_{\mathrm{d}}\right), \\
\varepsilon \frac{\partial \mathrm{N}}{\partial \mathrm{t}}+\nabla \cdot\left(\mathrm{N} \overrightarrow{\mathrm{q}}_{\mathrm{d}}\right)=0 .
\end{gathered}
$$

Let $\mathrm{C}_{\mathrm{v}}, \mathrm{C}_{\mathrm{p}}, \mathrm{C}_{\mathrm{pt}}, \mathrm{T}, \mathrm{C}$ and $\mathrm{k}$ denote respectively the heat capacity of fluid at constant volume, heat capacity of fluid at constant pressure, heat capacity of particles, temperature, solute concentration and "effective thermal conductivity" of the fluid. Let $\mathrm{C}_{\mathrm{v}}^{\prime}, \mathrm{C}_{\mathrm{pt}}^{\prime}$ and $\mathrm{k}^{\prime}$ denote the analogous solute coefficients. When particles and the fluid are in thermal and solute equilibrium, the equations of heat and solute conduction give

$$
\begin{gathered}
{\left[\rho C_{v} \varepsilon+\rho_{S} C_{S}(1-\varepsilon)\right] \frac{\partial T}{\partial t}+\rho C_{v}(\vec{q} \cdot \nabla) T} \\
+m N C_{p t}\left(\varepsilon \frac{\partial}{\partial t}+\vec{q}_{d} \cdot \nabla\right) T=k \nabla^{2} T, \\
{\left[\rho C_{v}^{\prime} \varepsilon+\rho_{S} C_{S}^{\prime}(1-\varepsilon)\right] \frac{\partial C}{\partial t}+\rho C_{v}^{\prime}(\vec{q} \cdot \nabla) C+m N C_{p t}^{\prime}\left(\varepsilon \frac{\partial}{\partial t}+\vec{q}_{d} \cdot \nabla\right) C=k^{\prime} \nabla^{2} C}
\end{gathered}
$$


where $\rho_{\mathrm{S}}, \mathrm{C}_{\mathrm{S}}$ are the density and heat capacity of the solid matrix, respectively.

Spiegel and Veronis[12] have expressed any state variable (pressure, density or temperature), say $\mathrm{X}$, in the form

$$
X=X_{m}+X_{o}(z)+X^{\prime}(x, y, z, t),
$$

where $X_{\mathrm{m}}$ stands for the constant space distribution of $\mathrm{X}, \mathrm{X}_{\mathrm{O}}$ is the variation in $\mathrm{X}$ in the absence of motion, and $\mathrm{X}^{\prime}(\mathrm{x}, \mathrm{y}, \mathrm{z}, \mathrm{t})$ stands for the fluctuations in $\mathrm{X}$ due to the motion of the fluid. Following Spiegel and Veronis[12], we have

$$
\begin{gathered}
T(z)=-\beta z+T_{0}, \\
p(z)=p_{m}-g \int_{0}^{z}\left(\rho_{m}+\rho_{0}\right) d z, \\
\rho(x)=\rho_{m}\left[1-\alpha\left(T-T_{m}\right)+\alpha^{\prime}\left(C-C_{m}\right)+\alpha^{\prime \prime}\left(p-p_{m}\right)\right], \\
\alpha=-\left(\frac{1}{\rho} \frac{\partial \rho}{\partial T}\right), \quad \alpha^{\prime}=\left(\frac{1}{\rho} \frac{\partial \rho}{\partial C}\right), \quad \alpha^{\prime \prime}=\left(\frac{1}{\rho} \frac{\partial \rho}{\partial p}\right) .
\end{gathered}
$$

Here $\mathrm{p}_{\mathrm{m}}, \rho_{\mathrm{m}}$ stand for the space distribution of $\mathrm{p}$ and $\rho$, and $\mathrm{T}_{0}, \rho_{0}$ stand for the temperature and density of the fluid at the lower boundary (and in the absence of motion).

Since density variations are mainly due to variations in temperature and solute concentrations, equations (1)-(6) must be supplemented by the equation of state

$$
\rho(z)=\rho_{m}\left[1-\alpha\left(T-T_{m}\right)+\alpha^{\prime}\left(C-C_{m}\right)\right] .
$$

Let $\delta \rho, \delta \mathrm{p}, \theta, \gamma, \overrightarrow{\mathrm{q}}, \overrightarrow{\mathrm{q}}_{\mathrm{d}}$ and $\mathrm{N}$ denote the perturbations in fluid density $\rho$, pressure $\mathrm{p}$, temperature $\mathrm{T}$, solute concentration $\mathrm{C}$, fluid velocity $(0,0,0)$, particles velocity $(0,0,0)$ and particle number density $\mathrm{N}_{0}$, respectively. Then the linearized perturbation equations, under the Spiegel and Veronis [12] assumptions, are

$$
\begin{gathered}
\frac{1}{\varepsilon} \frac{\partial \vec{q}}{\partial t}=-\frac{1}{\rho_{m}} \nabla \delta p-g\left(\frac{\delta \rho}{\rho_{m}}\right) \vec{\lambda}_{1} \\
+\left(v+v^{\prime} \frac{\partial}{\partial t}\right)\left(\frac{1}{\varepsilon} \nabla^{2}-\frac{1}{k_{1}}\right) \vec{q}+\frac{K N_{0}}{\rho_{m} \varepsilon}\left(\vec{q}_{d}-\vec{q}\right), \\
\nabla \cdot \vec{q}=0, \\
m N_{0} \frac{\partial \vec{q}_{d}}{\partial t}=K N_{0}\left(\vec{q}-\vec{q}_{d}\right), \\
\varepsilon \frac{\partial N}{\partial t}+\nabla \cdot\left(N_{0} \vec{q}_{d}\right)=0, \\
(E+h \varepsilon) \frac{\partial \theta}{\partial t}=\left(\beta-\frac{g}{C_{p}}\right)(w+h s)+\kappa \nabla^{2} \theta, \\
\left(E^{\prime}+h^{\prime} \varepsilon\right) \frac{\partial \gamma}{\partial t}=\beta^{\prime}\left(w+h^{\prime} s\right)+\kappa^{\prime} \nabla^{2} \gamma .
\end{gathered}
$$

Here

$$
\begin{aligned}
& E=\varepsilon+(1-\varepsilon) \frac{\rho_{S} C_{S}}{\rho_{m} C_{v}}, E^{\prime}=\varepsilon+(1-\varepsilon) \frac{\rho_{S} C_{S}^{\prime}}{\rho_{m} C_{v}^{\prime}}, \\
& h=\frac{f C_{p t}}{C_{v}}, h^{\prime}=\frac{f C_{p t}^{\prime}}{C_{v}^{\prime}}, f=\frac{m N_{0}}{\rho_{m}}, \kappa=\frac{k}{\rho_{m} C_{v}}, \kappa^{\prime}=\frac{k^{\prime}}{\rho_{m} C_{v}^{\prime}} \\
& \text { and } \delta \rho=-\rho_{m}\left(\alpha \theta-\alpha^{\prime} \gamma\right) .
\end{aligned}
$$

Using $d, d^{2} / \kappa, \kappa / d, \rho v \kappa / d^{2}, \beta d$ and $\beta^{\prime} d$ to denote the length, time, velocity, pressure, temperature and solute concentration scale factors, respectively, the linearized perturbation equations become

$$
\begin{gathered}
p_{1}^{-1} \frac{\partial \vec{q}^{*}}{\partial t^{*}}=-\nabla^{*} \delta p^{*}+R \theta^{*} \vec{\lambda}_{1}-S \gamma^{*} \vec{\lambda}_{1} \\
+\left(1+A \frac{\partial}{\partial t^{*}}\left(\frac{1}{\varepsilon} \nabla^{* 2}-\frac{1}{P}\right) \vec{q}^{*}+\omega\left(\vec{q}_{d}^{*}-\vec{q}^{*}\right),\right. \\
\nabla^{*} \cdot \vec{q}^{*}=0, \\
\left(\tau \frac{\partial}{\partial t^{*}}+1\right) \vec{q}_{d}^{*}=\vec{q}^{*}, \\
\left(\frac{\partial M}{\partial t^{*}}+\nabla^{*} \cdot \vec{q}_{d}^{*}\right)=0, \\
(E+h \varepsilon) \frac{\partial \theta^{*}}{\partial t^{*}}=\frac{G-1}{G}\left(w^{*}+h s^{*}\right)+\nabla^{* 2} \theta^{*}, \\
\left(E^{\prime}+h^{\prime} \varepsilon\right) \frac{\partial \gamma^{*}}{\partial t^{*}}=\left(w^{*}+h^{\prime} s^{*}\right)+\frac{1}{\lambda} \nabla^{* 2} \gamma^{*},
\end{gathered}
$$

where

$$
\begin{aligned}
& P=\frac{k_{1}}{d^{2}}, G=\frac{C_{p} \beta}{g}, p_{1}=\frac{\varepsilon v}{\kappa}, R=\frac{g \alpha \beta d^{4}}{\nu \kappa}, S=\frac{g \alpha^{\prime} \beta^{\prime} d^{4}}{v \kappa^{\prime}}, M=\frac{\varepsilon N}{N_{0}}, \\
& \omega=\frac{K N_{0} d^{2}}{\rho_{m^{2}} v}, \tau=\frac{m \kappa}{K d^{2}}, f=\frac{m N_{0}}{\rho_{m}}=\tau \omega p, A=\left(\frac{\nu^{\prime}}{v}\right) \frac{\kappa}{d^{2}} \text { and } \lambda=\frac{\kappa}{\kappa^{\prime}},
\end{aligned}
$$

and starred $(*)$ quantities are expressed in dimensionless form. Hereafter, we suppress the stars for convenience.

Eliminating $\vec{q}_{d}$ from equation (15) with the help of equation (17) and then eliminating $\mathrm{u}, \mathrm{v}, \delta \mathrm{p}$ from the three scalar equations of (15), and using (16), we obtain

$$
\begin{gathered}
{\left[L_{1}-L_{2}\left(1+A \frac{\partial}{\partial t}\right)\left(\frac{1}{\varepsilon} \nabla^{2}-\frac{1}{P}\right)\right] \nabla^{2} w=L_{2}\left(R \nabla_{1}^{2} \theta-S \nabla_{1}^{2} \gamma\right),} \\
L_{2}\left[(E+h \varepsilon) \frac{\partial}{\partial t}-\nabla^{2}\right] \theta=\left(\frac{G-1}{G}\right)\left(\tau \frac{\partial}{\partial t}+H\right) w, \\
L_{2}\left[\left(E^{\prime}+h^{\prime} \varepsilon\right) \frac{\partial}{\partial t}-\frac{1}{\lambda} \nabla^{2}\right] \gamma=\left(\tau \frac{\partial}{\partial t}+H^{\prime}\right) w
\end{gathered}
$$

where

$$
\begin{aligned}
& L_{1}=p_{1}^{-1}\left(\tau \frac{\partial^{2}}{\partial t^{2}}+F \frac{\partial}{\partial t}\right) \\
& L_{2}=\left(\tau \frac{\partial}{\partial t}+1\right), \nabla_{1}^{2}=\frac{\partial^{2}}{\partial x^{2}}+\frac{\partial^{2}}{\partial y^{2}}, \\
& \nabla^{2}=\frac{\partial^{2}}{\partial x^{2}}+\frac{\partial^{2}}{\partial y^{2}}+\frac{\partial^{2}}{\partial z^{2}}, \\
& F=f+1, H=h+1, H^{\prime}=h^{\prime}+1 .
\end{aligned}
$$

\section{Dispersion Relation}

Decomposing the perturbations into normal modes by seeking solutions in the form of functions of $x, y, t$

$$
[w, \theta, \gamma]=[W(z), \Theta(z), \Gamma(z)] \exp \left(i k_{x} x+i k_{y} y+n t\right),
$$

where $n$ is, in general, complex, and $k=\left(k_{x}^{2}+k_{y}^{2}\right)^{1 / 2}$ is the wave number of disturbance.

Eliminating $\theta$ and $\gamma$ between equations (21)-(23) and using expression (24), we obtain

$$
\begin{aligned}
& {\left[L_{1}+\frac{L_{2}}{P}(1+A n)-\frac{L_{2}}{\varepsilon}(1-A n)\left(D^{2}-k^{2}\right)\right]} \\
& {\left[D^{2}-k^{2}-n(E+h \varepsilon)\right]\left[D^{2}-k^{2}-\lambda n\left(E^{\prime}+h^{\prime} \varepsilon\right)\right]\left(D^{2}-k^{2}\right) W} \\
& =\left(\frac{G-1}{G}\right)(\tau n+H) R k^{2}\left[D^{2}-k^{2}-\lambda n\left(E^{\prime}+h^{\prime} \varepsilon\right)\right] W \\
& -\lambda\left(\tau n+H^{\prime}\right) S k^{2}\left[D^{2}-k^{2}-n(E+h \varepsilon)\right] W
\end{aligned}
$$


where

$$
L_{1}=p_{1}^{-1}\left(\tau n^{2}+F n\right), L_{2}=\tau n+1 \text { and } D=\frac{d}{d z} .
$$

\section{The Stationary Convection}

When the instability sets in as stationary convection, the marginal state will be characterized by $n=0$. Putting $n=0$, the dispersion relation $(25)$ reduces to

$$
\left[\frac{1}{P}-\frac{1}{\varepsilon}\left(D^{2}-k^{2}\right)\right]\left(D^{2}-k^{2}\right)^{2} W=\left(\frac{G}{G-1}\right) k^{2} R H W-\lambda k^{2} S H^{\prime} W .
$$

We thus find that for stationary convection the viscoelastic parameter A vanishes with $\mathrm{n}$ and Rivlin-Ericksen viscoelastic fluid behaves like an ordinary Newtonian fluid.

Considering the case of two free boundaries, it can be shown that all the even order derivatives of $\mathrm{W}$ vanish on the boundaries and hence the proper solution of equation (26) characterizing the lowest mode is

$$
W=W_{0} \sin \pi z
$$

where $\mathrm{W}_{0}$ is a constant. Substituting the solution (27) in equation (26), we obtain

$$
R=\frac{\left(\frac{G}{G-1}\right)\left[\left(\frac{1}{P}+\frac{\pi^{2}+k^{2}}{\varepsilon}\right)\left(\pi^{2}+k^{2}\right)^{2}+\lambda k^{2} H^{\prime} S\right]}{k^{2} H} .
$$

If $\mathrm{R}_{\mathrm{C}}$ denotes the critical Rayleigh number in the $\mathrm{ab}$ sence of compressibility and $\overline{\mathrm{R}}_{\mathrm{C}}$ stands for the critical Rayleigh number in the presence of compressibility, then we find that

$$
\bar{R}_{C}=\left(\frac{G}{G-1}\right) R_{C} .
$$

Since critical Rayleigh number is positive and finite, so $\mathrm{G}>1$ and we obtain a stabilizing effect of compressibility as its result is to postpone the onset of thermosolutal convection in a fluid-particle layer of porous medium.

To study the effect of medium permeability, suspended particles and stable solute gradient, we examine the nature of $\frac{d R}{d P}, \frac{d R}{d H}$ and $\frac{d R}{d S}$ analytically.

Equation (28) yields

$$
\begin{gathered}
\frac{d R}{d P}=-\left(\frac{G}{G-1}\right) \frac{\left(\pi^{2}+k^{2}\right)^{2}}{k^{2} H P^{2}}, \\
\frac{d R}{d H}=-\left(\frac{G}{G-1}\right) \frac{\left(\frac{1}{P}+\frac{\pi^{2}+k^{2}}{\varepsilon}\right)\left(\pi^{2}+k^{2}\right)^{2}+\lambda k^{2} H^{\prime} S}{k^{2} H^{2}},
\end{gathered}
$$

and

$$
\frac{d R}{d S}=\lambda\left(\frac{G}{G-1}\right) \frac{H^{\prime}}{H} .
$$

It is clear from equations (29)-(31) that for stationary convection the medium permeability and suspended particles hasten the onset of convection whereas the stable solute gradient postpones the onset of convection in Rivlin-Ericksen compressible viscoelastic fluid permeated with suspended particles, heated and soluted from below in porous medium.

We now examine the dispersion relation (28) numerically.
We have plotted the Rayleigh number versus the medium permeability $\mathrm{P}$, suspended particles parameter $\mathrm{H}$ and stable solute gradient $\mathrm{S}$ in Figures 1-3, respectively.

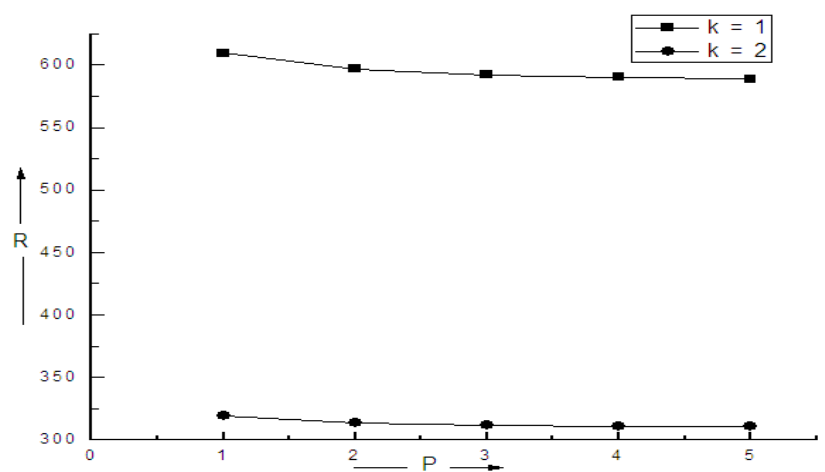

Figure 1. The variation of Rayleigh number $(\mathrm{R})$ with medium permeability (P) for fixed values of $\mathrm{G}=10, \mathrm{H}=0.5, \mathrm{~g}=0.5$ and $\mathrm{k}=1.2$

In Figure 1, the Rayleigh number $\mathrm{R}$ is plotted against medium permeability $\mathrm{P}$ for fixed values of $G=10, H^{\prime}=5$, $\lambda=1, S=10, H=5, \varepsilon=0.5$ and $k=1,2$. As the value of medium permeability increases, the corresponding value of the Rayleigh number decreases, showing its destabilizing effect on the system.

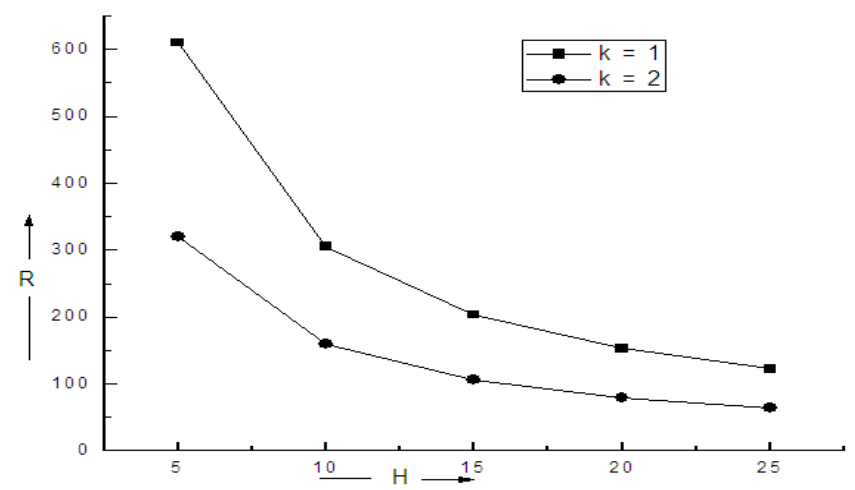

Figure 2. The variation of Rayleigh number $(\mathrm{R})$ with suspended particles $(\mathrm{H})$ for fixed values of $\mathrm{G}=10, \mathrm{H}=0.5, \lambda=1, \mathrm{~S}=0.5$ and $\mathrm{k}=1.2$

In Figure 2, the Rayleigh number $\mathrm{R}$ is plotted against suspended particles parameter $\mathrm{H}$ for fixed values of $\mathrm{G}=10, \quad \mathrm{P}=1, \mathrm{H}^{\prime}=5, \lambda=1, \mathrm{~S}=10, \varepsilon=0.5$ and $\mathrm{k}=1,2$. The Rayleigh number decreases with the increase in the suspended particles parameter showing its destabilizing effect on the system.

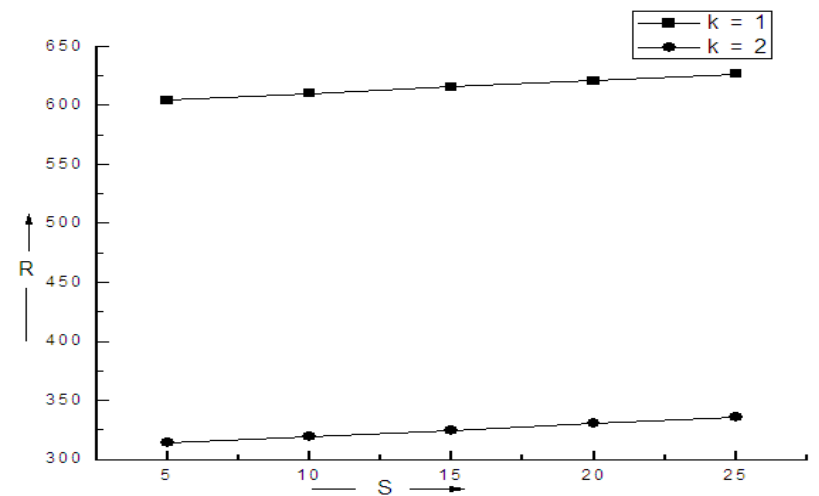

Figure 3. The variation of Rayleigh number $(\mathrm{R})$ with stable solute gradient (S) for fixed values of $\mathrm{G}=10, \mathrm{P}=1, \mathrm{H}^{1}=5, \lambda=1, \mathrm{H}=5, \varepsilon=0.5$ and $\mathrm{k}=1.2$ 
Figure 3 shows the variation of $\mathrm{R}$ with respect to stable solute gradient $\mathrm{S}$ for fixed values of $\mathrm{G}=10, \mathrm{P}=1, \mathrm{H}^{\prime}=5$, $\lambda=1, \mathrm{H}=5, \varepsilon=0.5$ and $\mathrm{k}=1,2$. The Rayleigh number $\mathrm{R}$ increases with an increase in stable solute gradient parameter $\mathrm{S}$, showing its stabilizing effect on the system.

Thus, the medium permeability and suspended particles parameter have destabilizing effect whereas stable solute gradient has stabilizing effect on Rivlin-Ericksen compressible viscoelastic fluid permeated with suspended particles, heated and soluted from below in porous medium.

\section{Principle of Exchange of Stabilities and Oscillatory Modes}

Let

$$
\begin{aligned}
U & =\left(D^{2}-k^{2}\right) W \text { and } \\
X & =\left[L_{1}+\frac{L_{2}}{P}(1+A n)-\frac{L_{2}}{\varepsilon}(1+A n)\left(D^{2}-k^{2}\right)\right] U .
\end{aligned}
$$

In terms of $X$, the equation satisfied by $\mathrm{W}$ [cf. equation $(25)]$ is

$$
\begin{aligned}
& {\left[D^{2}-k^{2}-n(E+h \varepsilon)\right]\left[D^{2}-k^{2}-\lambda n\left(E^{\prime}+h^{\prime} \varepsilon\right)\right] X} \\
& =k^{2}\left(\frac{G-1}{G}\right) R(\tau n+H)\left[D^{2}-k^{2}-\lambda n\left(E^{\prime}+h^{\prime} \varepsilon\right)\right] \\
& W-\lambda k^{2} S\left(\tau n+H^{\prime}\right) \\
& {\left[D^{2}-k^{2}-n(E+h \varepsilon)\right] W .}
\end{aligned}
$$

Here we consider the case of two free surfaces having uniform temperature and solute concentration. The boundary conditions appropriate for the problem are

$$
W=D^{2} W=0, \Theta=0, \mathrm{~T}=0 \text { at } z=0 \text { and } 1 .
$$

Multiplying equation (33) by $\mathrm{X}^{*}$, the complex conjugate of $\mathrm{X}$, integrating over the range of $\mathrm{z}$ and using the boundary conditions (34), we get

$$
\begin{aligned}
& I_{1}+n\left[(E+h \varepsilon)+\lambda\left(E^{\prime}+h^{\prime} \varepsilon\right)\right] I_{2}+\lambda n^{2}(E+h \varepsilon)\left(E^{\prime}+h^{\prime} \varepsilon\right) I_{3} \\
& =k^{2}\left(\frac{G-1}{G}\right) R(\tau n+H)\left(L_{1}^{*}+\frac{L_{2}^{*}}{P}(1+A n)\right)\left[I_{4}+\lambda n\left(E^{\prime}+h^{\prime} \varepsilon\right) I_{5}\right]-\lambda k^{2} S\left(\tau n+H^{\prime}\right) \\
& \left(L_{1}^{*}+\frac{L_{2}^{*}}{P}(1+A n)\right)\left[I_{4}+\lambda n\left(E+h^{\prime} \varepsilon\right) I_{5}\right]+k^{2} \frac{L_{2}^{*}}{\varepsilon}\left[\left(\frac{G-1}{G}\right) R(\tau n+H)-\lambda S\left(\tau n+H^{\prime}\right)\right] I_{6} \\
& +k^{2} \lambda n \frac{L_{2}^{*}}{\varepsilon}(1+A n)\left[\left(\frac{G-1}{G}\right) R(\tau n+H)\left(E^{\prime}+h^{\prime} \varepsilon\right)-S\left(\tau n+H^{\prime}\right)(E+h \varepsilon)\right] I_{7},
\end{aligned}
$$

where

$$
\begin{gathered}
I_{1}=\int_{0}^{1}\left(\left|D^{2} X\right|^{2}+2 k^{2}|D X|^{2}+k^{4}|X|^{2}\right) d z, I_{2}=\int_{0}^{1}\left(|D X|^{2}+k^{2}|X|^{2}\right) d z, \\
I_{3}=\int_{0}^{1}\left(|X|^{2}\right) d z \\
I_{4}=\int_{0}^{1}\left(|U|^{2}\right) d z, I_{5}=\int_{0}^{1}\left(|D W|^{2}+k^{2}|W|^{2}\right) d z, I_{6}=\int_{0}^{1}\left(|D U|^{2}+k^{2}|U|^{2}\right) d z \\
I_{7}=\int_{0}^{1}\left(\left|D^{2} W\right|^{2}+2 k^{2}|D W|^{2}+k^{4}|W|^{2}\right) d z
\end{gathered}
$$

which are all positive definite.

Putting $n=i n_{0}$, where $n_{0}$ is real, into equation (35) and equating imaginary parts, we obtain

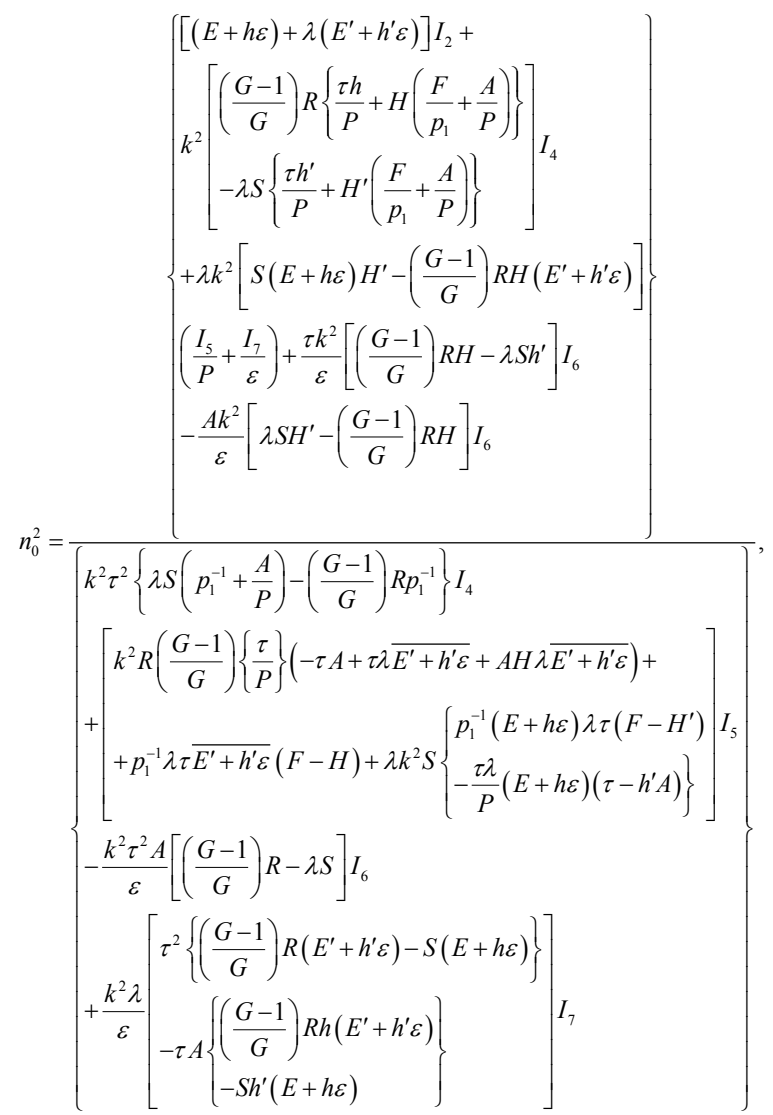

or

$$
n_{0}=0 \text {. }
$$

Equations (37) and (38) imply that $\mathrm{n}_{0} \neq 0$ or $\mathrm{n}_{0}=0$, which means that modes may be oscillatory or non-oscillatory. In the absence of stable solute gradient and viscoelastic parameter, equations (37) and (38) become

$$
n_{0}^{2}=-\frac{\left(\frac{G}{G-1}\right)(E+h \varepsilon) I_{2}+k^{2} R\left\{p_{1}^{-1} H F+\frac{\tau h}{P}\right\} I_{4}+\frac{k^{2} \tau}{\varepsilon} R h I_{6}}{k^{2} \tau^{2} R p_{1}^{-1} I_{4}}
$$

or

$$
n_{0}=0 \text {. }
$$

Since the integrals are positive definite and $n_{0}$ is real, it follows that $n_{0}=0$ and the principle of exchange of stabilities is satisfied, in the absence of stable solute gradient and viscoelastic parameter. In the presence of stable solute gradient and viscoelastic parameter, the principle of exchange of stabilities is not satisfied and oscillatory modes come into play. Thus, the stable solute gradient and viscoelastic parameter, introduces oscillatory modes which were non-existent in their absence.

\section{Conclusions}


Pardeep Kumar et al.: Double-Diffusive Convection in Compressible Viscoelastic Dusty Fluid Through Brinkman Porous Media

The effect of suspended particles on the double-diffusive convection in a layer of compressible Rivlin-Ericksen viscoelastic fluid heated and soluted from below through porous medium is considered in the present paper. The investigation of double-diffusive convection is motivated by its complexities as a double diffusion phenomena as well as its direct relevance to geophysics and astrophysics. The main conclusions from the analysis of this paper are as follows:

(a) For the case of stationary convection the following observations are made:

(i) The viscoelastic parameter vanishes with $n$ and Rivlin-Ericksen viscoelastic fluid behaves like an ordinary Newtonian fluid.

(ii) The stable solute gradient and compressibility have stabilizing effect on the system.

(iii) The suspended particles and medium permeability have destabilizing effect on the system.

(b) It is also observed from Figures 1-3 that suspended particles and medium permeability have destabilizing effect whereas stable solute gradient has stabilizing effect on the system.

(c) It is observed that stable solute gradient and viscoelastic parameter introduce oscillatory modes in the system, which was non-existent in their absence.

(d) In the absence of stable solute gradient and viscoelastic parameter, oscillatory modes are not allowed and the principle of exchange of stabilities is valid.

\section{REFERENCES}

[1] Chandrasekhar, S.: Hydrodynamic and Hydromagnetic Stability, Clarendon Press, Oxford, UK, 1961

[2] Veronis, G.: On finite amplitude instability in thermohaline convection, J. Marine Research, 23, pp. 1-17, 1965

[3] Lister, P.F.: On the thermal balance of a mid-ocean ridge, Geophysical J. Royal Astronomical Soc., 26, pp. 515-535, 1972

[4] Beavers, G.S., Sparrow, E.M. and Magnuson, R.A.: Experiments on coupled parallel flows in a channels and a bounding porous medium, J. Basic Engng. Trans. ASME, D92, pp. $843-851,1970$

[5] Joseph, D.D. and Tao, L.N.: The effect of permeability on the slow motion of a porous sphere in a viscous liquid, Zeit. Angew. Math. Mech., 44, pp. 361-365, 1964
[6] Stommel, H. and Fedorov, K.N.: Small scale structure in temperature and salinity near timor and mindano, Tellus, 19, p. 306,1967

[7] Linden, P.F.: Salt fingers in a steady shear flow, Geophys. Fluid Dynamics, 6, p. 1, 1974

[8] Brakke, M.K.: Zone electrophoresis of dyes, proteins and viruses in density-gradient columns of sucrose solutions, Arch. Biochem. Biophysics, 55, pp.175-190, 1955

[9] Nason, P., Schumaker, V., Halsall, B. and Schwedes, J.: Formation of a streaming convective disturbance which may occur at one gravity during preparation of samples for zone centrifugation, Biopolymers, 7, pp.241-249, 1969

[10] Scanlon, J.W. and Segel, L.A.: Some effects of suspended particles on the onset of Be'nard convection, Phys. Fluids, 16, pp. 1573-1578, 1973

[11] Palaniswamy, V.I. and Purushotham, C.M., Stability of shear flow of stratified fluids with fine dust, Phys. Fluids, 24, pp. 1224-1229, 1981

[12] Spiegel, E.A. and Veronis, G.: On the Boussinesq approximation for a compressible fluid, Astrophys. J., 131, p. 442, 1960

[13] Bhatia, P.K. and Steiner, J.M.: Convective instability in a rotating viscoelastic fluid layer, Z. Angew. Math. Mech., 52, pp. $321-324,1972$

[14] Sharma. R.C.: Effect of rotation on thermal instability of a viscoelastic fluid, Acta Physica Hung., 40, pp. 11-17, 1976

[15] Rivlin, R.S. and Ericksen, J.L., Stress-deformation relaxations for isotropic materials, J. Rat. Mech. Anal., 4, pp. $323-425,1955$

[16] Sharma, R.C. and Kumar, P., Effect of rotation on thermal instability in Rivlin-Ericksen elastico-viscous fluid, Z. Naturforch., 51a, pp. 821-824, 1996

[17] Sharma, R.C. and Kumar, P., Thermal instability in Rivlin-Ericksen elastico-viscous fluid in hydromagnetics, $\mathrm{Z}$. Naturforch., 52a, pp. 369-371, 1997

[18] Sharma, R.C. and Kumar, P., Hydrodynamic stability of Rivlin - Ericksen elastico - viscous superposed conducting fluids, Z. Naturforch., 52a, pp. 528-532, 1997

[19] Kumar, P. and Singh, G.J., Stability of two superposed Rivlin-Ericksen viscoelastic fluids in the presence of suspended particles, Rom. J. Phys., 51,pp. 927-935, 2006

[20] Kumar, P., Lal, R. and Singh, M., Hydrodynamic and hydromagnetic stability of two stratified Rivlin-Ericksen elastico-viscous superposed fluid, Int. J. Appl. Mech. Engng., 12, pp. 645-653, 2007 